\title{
Mapeo geoepidemiológico en la nueva vigilancia de salud pública. El caso de la malaria en Chiapas, México, en 2002
}

\author{
Carlos Castillo-Salgado
}

Department of Epidemiology, Bloomberg School of Public Health, Baltimore, MD, USA

\section{Resumen}

La nueva vigilancia en salud pública ha demando, en los ámbitos global, nacional y local, el uso de nuevos métodos y herramientas analíticas que permitan un mejor reconocimiento del comportamiento epidemiológico de los eventos y riesgos a la salud poblacional. La identificación de eventos prioritarios en tiempo y espacio es de importancia fundamental para la descripción espacial de la situación de enfermedades y eventos prioritarios de salud, ya que facilita la identificación de importantes factores de riesgo sociales, ambientales y relacionados con la atención a la salud. Esta investigación examina la aplicación y la utilización de herramientas geoespaciales de los sistemas de información geográfica (SIG) que permitan la identificación de conglomerados espaciales y epidemiológicos relevantes de la malaria en Chiapas, México. El diseño de estudio fue de carácter ecológico y el nivel de agregación de la información de las variables epidemiológicas y espaciales fue el de municipios y ciudades. Los datos fueron recolectados en todos municipios del estado de Chiapas durante los años 2000-2002. Las variables principales fueron el número de casos y el tipo de malaria diagnosticada por gota gruesa de los informes semanales. Así mismo, se incluyeron la edad, el sexo, la etnicidad, el nivel de alfabetización de los casos de malaria y factores ambientales como la altitud y la red y el tipo de carreteras en municipios y ciudades. Se pudo visualizar mediante los mapas temáticos y las herramientas analíticas de los SIG que importantes factores de riesgo poblacional asociados a la malaria en Chiapas fueron la baja tasa de alfabetización, poblaciones con alto porcentaje de población indígena que refleja las desigualdades sociales en salud y la gran carga de enfermedad que persisten en este importante grupo vulnerable. La presencia de la red de carreteras permitió una mayor difusión espacial de la malaria. Un importante conglomerado epiespacial fue identificado en las áreas y la población próximas a la frontera sur. El uso de los SIG en los niveles locales asiste a la caracterización de la estratificación epidemiológica de la malaria y a la focalización efectiva y equitativa de las acciones de prevención y control de la malaria.

PALABRAS CLAVE: Malaria. Vigilancia en salud pública. Estratificación epidemiológica de la malaria. Sistemas. Métodos geoespaciales. Índices de autocorrelación espacial.

\begin{abstract}
The new public health surveillance requires at the global, national and local levels the use of new authoritative analytical approaches and tools for better recognition of the epidemiologic characteristics of the priority health events and risk factors affecting the population health. The identification of the events in time and space is of fundamental importance so that the geo-spatial description of the situation of diseases and health events facilitates the identification of social, environmental and health care related risks. This assessment examines the application and use of geo-spatial tools for identifying relevant spatial and epidemiological conglomerates of malaria in Chiapas, Mexico. The study design was ecological and the level of aggregation of the collected information of the epidemiological and spatial variables was municipalities. The data were collected in all municipalities of the state of Chiapas, Mexico during the years 2000-2002. The main outcome variable was cases and types of malaria diagnosed by blood smears in weekly reports. Independent variables were age, sex, ethnicity, literacy of the cases
\end{abstract}

Correspondencia:

Carlos Castillo-Salgado

615 N. Wolfe Street, Room E-6136

Baltimore, MD 21205

E-mail: Ccastil3@jhu.edu
Fecha de recepción: 07-08-2017

Fecha de aceptación: 07-09-2017

DOI://dx.doi.org/10.24875/GMM.M17000001
Gac Med Mex. 2017;153;Sup 2:S5-S12

Contents available at PubMed www.anmm.org.mx 
of malaria and environmental factors such as altitude, road type and network in the municipalities and cities of Chiapas. The production of thematic maps and the application of geo-spatial analytical tools such Moran and local indicator of spatial autocorrelation metrics for malaria clustering allowed the visualization and recognition that the important population risk factors associated with high malaria incidence in Chiapas were low literacy rate, areas with high percentage of indigenous population that reflects the social inequalities gaps in health and the great burden of disease that is affecting this important vulnerable group in Chiapas. The presence of road networks allowed greater spatial diffusion of Malaria. An important epidemiological and spatial cluster of malaria was identified in the areas and populations in the proximity of the southern border. The use of geospatial metrics in local areas will assist in the epidemiological stratification of malaria for better targeting more effective and equitable prevention and control interventions.

KEY WORDS: Malaria. Public health surveillance. Epidemiological stratification of malaria. Geospatial methods. Indices of spatial autocorrelation.

\section{Introducción}

Esta investigación examina la aplicación y la utilización de los métodos y métricas geoespaciales ${ }^{1-4}$ como nuevas herramientas de la vigilancia en salud pública de problemas prioritarios como la malaria. Los análisis geoepidemiológicos permitieron una mejor caracterización e identificación de los factores poblacionales asociados a brotes y conglomerados geo-epidemiológicos de la transmisión de la malaria en Chiapas, México ${ }^{5-7}$.

La comunidad de la salud pública mundial ha reconocido a la «vigilancia en salud pública» como una de las más importantes funciones y competencias de la salud pública ${ }^{1,8,9}$.

El desarrollo de los sistemas de información geográfica (SIG) y su aplicación a la salud pública y la epidemiología han permitido un gran avance en los análisis epidemiológicos requeridos para la prevención y el control de las enfermedades prioritarias ${ }^{2-3,10}$. Con esta importante incorporación metodológica, la epidemiología pudo integrar de manera simultánea las variables fundamentales de población, tiempo y lugar (geografía). Previamente, la mayoría de las investigaciones operacionales de la epidemiología se concentraban en los modelos multivariados de persona-tiempo. Sin embargo, con la nueva visión de incorporar la dimensión y variables espaciales en los análisis de situación de salud se desarrollaron diversos métodos geoespaciales y nuevas métricas de gran valor para el reconocimiento de la presencia de conglomerados epiespaciales de alto riesgo para la salud de la población y para el estudio de brotes epidémicos de los problemas de salud prioritarios $\mathrm{s}^{3,11,12}$.

La aplicación de los SIG en salud publica incluye el diseño, el desarrollo y la utilización de los métodos y herramientas de los SIG para la descripción de la situación de salud, la vigilancia en salud pública y los diversos análisis epidemiológicos que requiere la práctica de la salud pública ${ }^{10}$.

Recientemente, la Organización Mundial de la Salud $^{13,14}$ ha renovado su interés por el control y la erradicación de la malaria, solicitando herramientas efectivas para el abordaje de la situación de la malaria en los ámbitos locales, nacionales y globales. Así mismo, destacan iniciativas como la Agenda para la Investigación de la Erradicación de la Malaria ${ }^{15}$, que es una iniciativa financiada por la Fundación Bill y Melinda Gates dirigida a la reducción de la morbimortalidad de la malaria a través de su control, así como para identificar las brechas de conocimiento existente y las herramientas necesarias para la erradicación de la malaria $^{16}$.

Reconociendo esta renovación hacia el control y la erradicación de la malaria, es útil el considerar los aportes epidemiológicos de impacto operacional que ha tenido la estratificación epidemiológica de la malaria $^{17-19}$. Hemos caracterizado la estratificación epidemiológica de la malaria como una estrategia de focalización basada en un proceso continuo y sistemático de identificación y medición de los principales factores de riesgo locales que determinan la frecuencia, la distribución y la intensidad de la malaria, y de esta forma poder agrupar a las distintas áreas y poblaciones afectadas en estratos de riesgo epidemiológico semejantes debido a que comparten los principales factores de riesgo. Esto permite seleccionar y aplicar las intervenciones específicas de prevención y control diferenciadas para cada población, dejando atrás la visión de que una intervención única e igual para todas las poblaciones es suficiente. Este proceso permite optimizar la toma de decisiones, así como tener mayor efectividad y equidad en las intervenciones para la prevención y la eliminación de la malaria. Por otro lado, en los últimos años se ha incrementado enormemente la capacidad analítica de los SIG mediante métricas y 
procedimientos analíticos geoespaciales que permiten la identificación exitosa de conglomerados epidemiológicos y espaciales de problemas críticos de salud pública, como la malaria ${ }^{20-24}$.

\section{Métodos}

El diseño del estudio fue de carácter ecológico debido a que el nivel de agregación de la información de las variables epidemiológicas y espaciales fue el de municipios y ciudades. Los datos fueron recolectados en todos los municipios y ciudades del Estado de Chiapas, México, durante los años 2000-200225. Las variables principales fueron el número de casos y el tipo de malaria diagnosticada por gota gruesa de los informes semanales de vigilancia epidemiológica. Así mismo, se incluyeron la edad, el sexo, la etnicidad, el nivel de alfabetización de todos los casos de malaria, la altitud y la red y el tipo de carreteras en municipios y ciudades de Chiapas.

Se crearon diversas bases de datos epidemiológi$\cos$ (archivos de atributos) y datos cartográficos (archivo de fronteras *.shp) de todos los municipios y ciudades, así como de las carreteras (clasificadas por sectores) y de los niveles de elevación (mediante medición isométrica del territorio) de Chiapas. Se aplicaron estadísticas descriptivas de todas las variables, así como los análisis de correlación y concordancia de las mismas.

Para la caracterización de la presencia de conglomerados epidemiológicos utilizando pruebas de significación estadística se realizaron inicialmente las mediciones descriptivas (promedios, desviaciones estándar, tasas ajustadas por edad). Para las dos variables geográficas se hizo el cálculo del riesgo relativo para malaria. Para los análisis geoespaciales complejos se realizaron dos medidas de autocorrelación espacial.

Para la clasificación geoespacial y la aplicación de las métricas de autocorrelación espacial se utilizó el software de dominio público QGIS ${ }^{26}$. El índice de Moran global ${ }^{27}$ y el índice de Anselin local (LISA, Local Indicators of Spatial Association) ${ }^{28,29}$ fueron las dos métricas de autocorrelación espacial utilizadas para el reconocimiento de la presencia de conglomerados espaciales estadísticamente significativos. Así mismo, se utilizaron distintas herramientas analíticas de la aplicación informática GeoPublicHealth Plugin ${ }^{30}$, desarrolladas para el entrenamiento en epidemiología espacial de la Johns Hopkins's Bloomberg School of Public Health ${ }^{31}$.

\section{Resultados}

Debido a que los casos de malaria en Chiapas no tuvieron una distribución estadística normal o uniforme, el método de clasificación en los mapas fue de cortes naturales o de quintiles. La clasificación de distribución espacial de los casos de malaria fue de cortes naturales para describir la intensidad y la distribución de los casos y de las tasas ajustadas de malaria.

En la figura 1 se presenta un mapa que permite identificar los municipios y las ciudades donde se localizaron el mayor número de casos de malaria, usando un mapa temático de vigilancia tipo cloropleta. En los municipios, los casos de malaria se clasificaron en cinco rangos de 0 a 510 casos. Considerando la distribución de los casos de malaria, se seleccionaron los municipios con más de 74 casos de malaria. Como puede observarse en la figura 1, que contiene el mapa tipo cloropleta tanto de municipios como de ciudades, esto permitió la identificación de 14 municipios y dos ciudades con un número mayor de 74 casos de malaria. Al incluirse la capa de ciudades en el mapa se observó que las ciudades de Hidalgo y Tapachula tenían la mayor densidad de casos de malaria.

Se pudieron verificar los patrones de la distribución del número de casos, que fueron consistentes con la densidad poblacional en el área, pero ello no necesariamente era indicativo del riesgo epidemiológico, que podrá observarse en la figura 2.

En la figura 2 se presenta la distribución de la tasa de incidencia de malaria (incidencia parasitaria anual por 1000 habitantes). Reconociendo la importancia de conocer el análisis del riesgo epidemiológico de enfermar de malaria ${ }^{32-34}$, se calculó la incidencia de malaria por municipios y ciudades. El cálculo de la incidencia permitió reconocer y visualizar la intensidad del riesgo de enfermar de malaria en los distintos municipios y ciudades.

Como se esperaba, el mapeo de la incidencia de malaria mostró un patrón un poco distinto al que ofreció el mapa por número total de casos, tanto en las ciudades como en los municipios. El mapa de la tasa de incidencia muestra un cambio más gradual en las localidades adyacentes a las de mayor número de casos que el mostrado en el mapa del número de casos de malaria.

En la figura 3 puede observarse la incorporación en el análisis espacial de la dimensión de la altitud del suelo y de la red y el tipo de carreteras ${ }^{35,36}$ para 


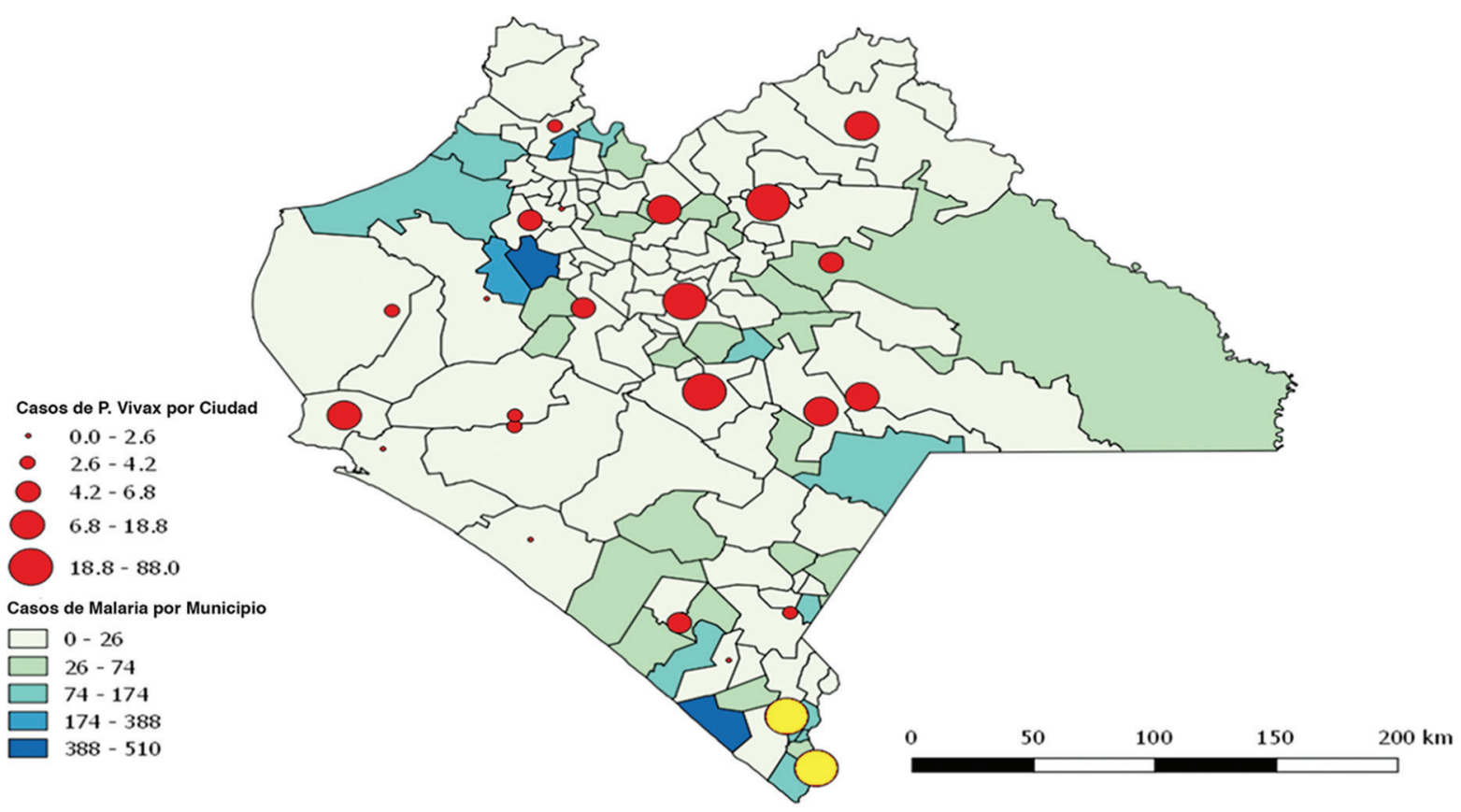

Figura 1. Número total de casos de malaria (Plasmodium vivax) por municipio y ciudades en Chiapas, México. La clasificación en rojo es del número de casos por ciudad, y la clasificación en azules es del número de casos por municipio. El remarcado en amarillo identifica a las ciudades que reportaron más de 74 casos de malaria. Se utilizaron simultáneamente fronteras geográficas de municipios y de ciudades para la creación de este mapa de cloropleta.

Incidencia de Malaria (IPA)

$0.0000-0.8492$

$0.8492-2.2751$

$2.2751-4.8469$

$4.8469-11.3472$

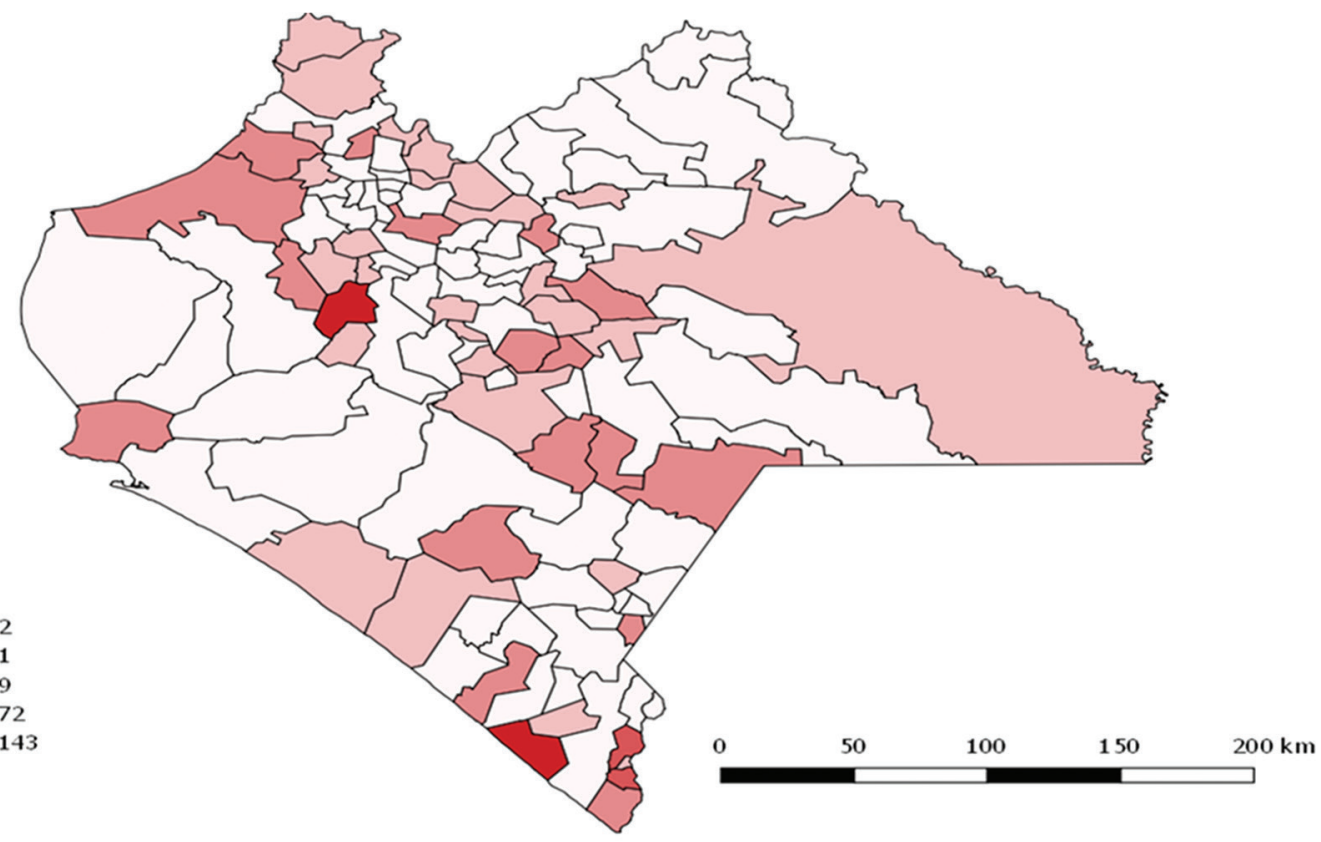

Figura 2. Tasa de incidencia de malaria (IPA: incidencia parasitaria anual) por 1000 habitantes y por municipio, en Chiapas, México. Cartografía de municipios y clasificación por la incidencia de malaria en Chiapas.

reconocer el papel que estas dos variables tuvieron en la intensidad de la transmisión de malaria (Plasmodium vivax), así como de la posible difusión espacial de la enfermedad.
La información de elevación y de las carreteras permitió identificar el patrón de difusión espacial que la malaria tuvo en los municipios, correspondiendo el mayor número de casos de malaria encontrado a la 


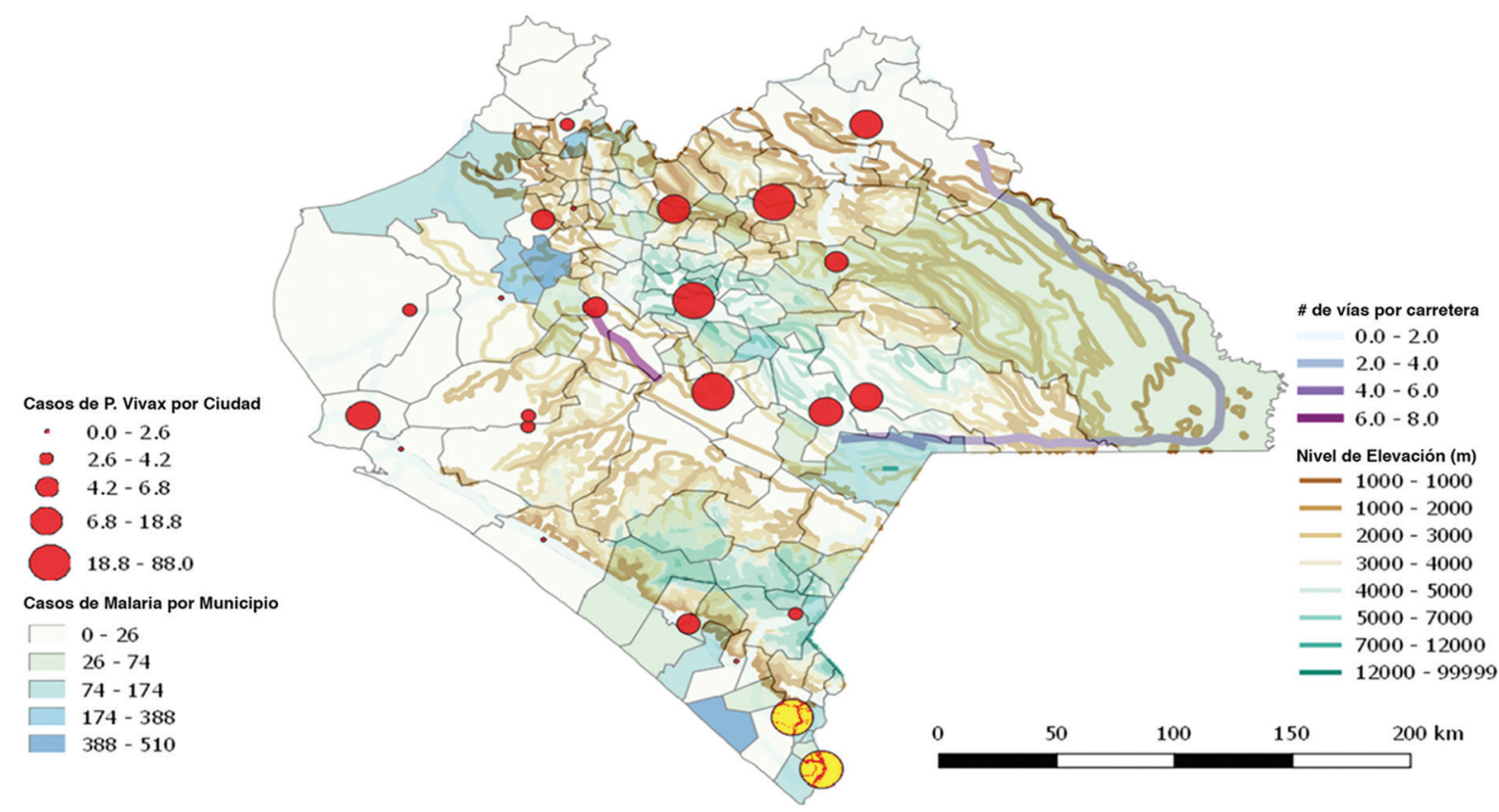

Figura 3. Número total de casos de malaria por municipio, ciudades, carreteras y elevación del suelo en Chiapas, México. Se agregaron a las variables de la figura 1 las variables espaciales de carreteras (por tipo) y de elevación del suelo.

línea de la costa del Pacífico y al interior del Estado en aquellos municipios localizados en la red de las carreteras principales.

Los municipios con mayor altitud mostraron una menor transmisión de la enfermedad esperada, debido probablemente a la existencia de un número menor de vectores (mosquitos), al igual que otros estudios que han reportado que el principal efecto de la altitud es la reducción del vector y la muy baja proporción de mosquitos infectados. Por tanto, se pudo observar en estos municipios la reducción de la diseminación de la malaria, como se mostró con el mapa de la elevación isométrica.

En las figuras 4 y 5 se visualiza la distribución de dos variables socioepidemiológicas de importancia poblacional ${ }^{37-39}$. La figura 4 muestra el nivel de alfabetización de los municipios de Chiapas, identificando a los de la costa, la selva y los preponderantemente con población indígena como los que tuvieron más bajos niveles de alfabetización, siendo este factor muy relevante para el incremento del riesgo de malaria y de otros importantes problemas de salud pública. La figura 5 muestra la distribución de la población indígena. Esta importante población continúa teniendo un elevado riesgo de malaria, así como manteniendo una gran vulnerabilidad en salud, y sigue habiendo elevados e injustos niveles de desigualdad en su salud y calidad de vida.

\section{Aplicación de métricas de autocorrelación espacial}

En este estudio se utilizó una de las métricas de autocorrelación espacial de mayor relevancia para la salud pública. Esta métrica, denominada LISA, fue desarrollada por Anselin ${ }^{29}$. Este índice permitió la identificación de «zonas calientes" (hot-spots) o de conglomerados espaciales de malaria que fueron estadísticamente significativos. También permitió la medición de la proximidad espacial en las áreas y poblaciones de Chiapas, y los riesgos poblacionales de la malaria. Esta herramienta pondera los puntos de mayor intensidad en la transmisión de la malaria y permite la detección de los conglomerados epidemiológicos existentes. Los patrones espaciales fueron consistentes con la agregación detectada en las áreas de alta incidencia de malaria.

Los patrones espaciales de la malaria o de otros problemas de salud no son debidos al azar, por lo que estos índices permiten analizar algunos de los predictores del riesgo de malaria, así como identificar áreas y conglomerados con mayores probabilidades de desarrollar la enfermedad en los grupos poblacionales que radican en dichas localidades o municipios. La premisa que revisar fue identificar la distribución del índice LISA y visualizar los conglomerados de la malaria en la región sureste de las poblaciones indígenas. 


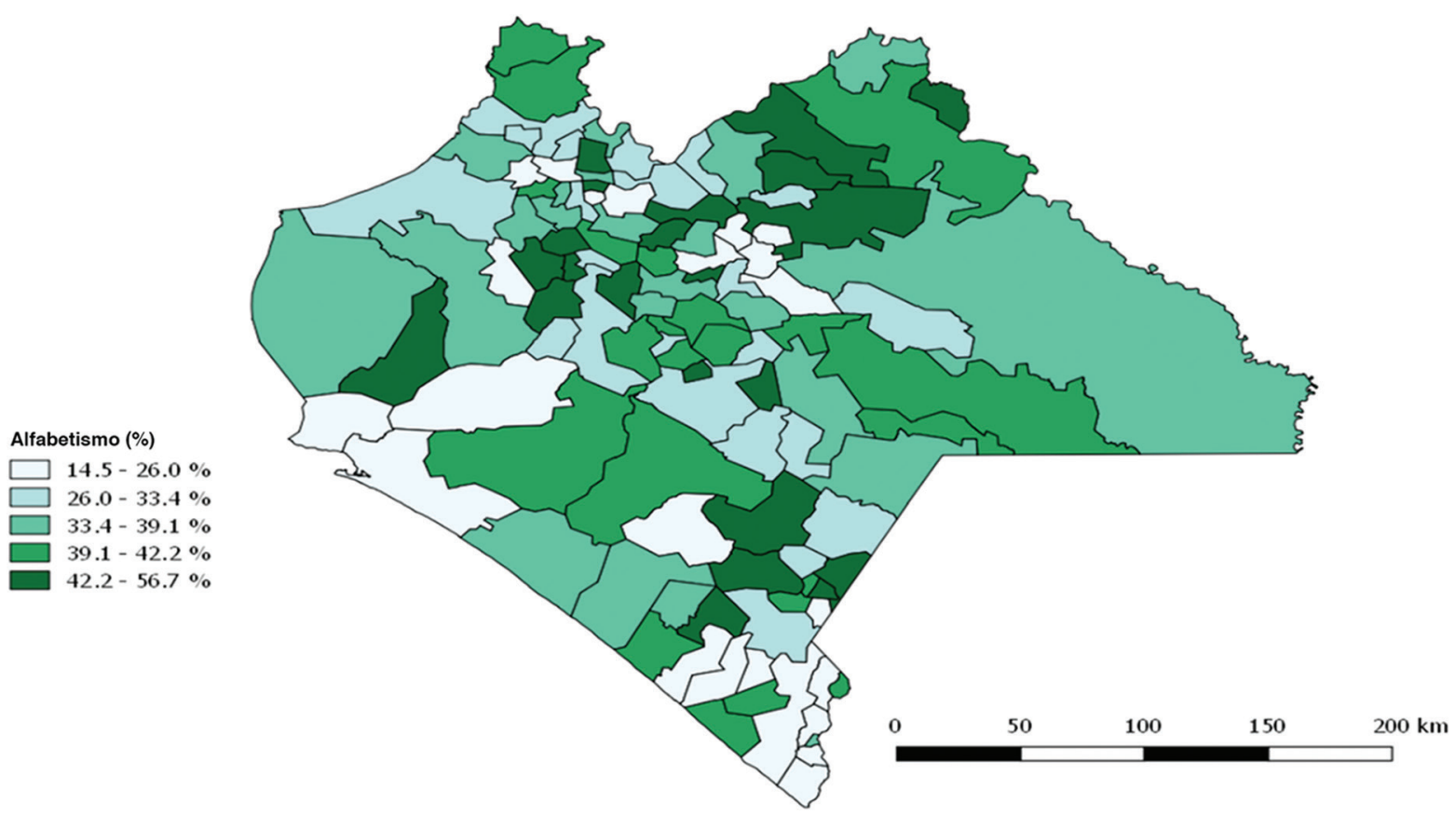

Figura 4. Mapa de la distribución de la población por nivel de alfabetización por municipio, en Chiapas. Cartografía de municipios y clasificación por quintiles del nivel de alfabetización.
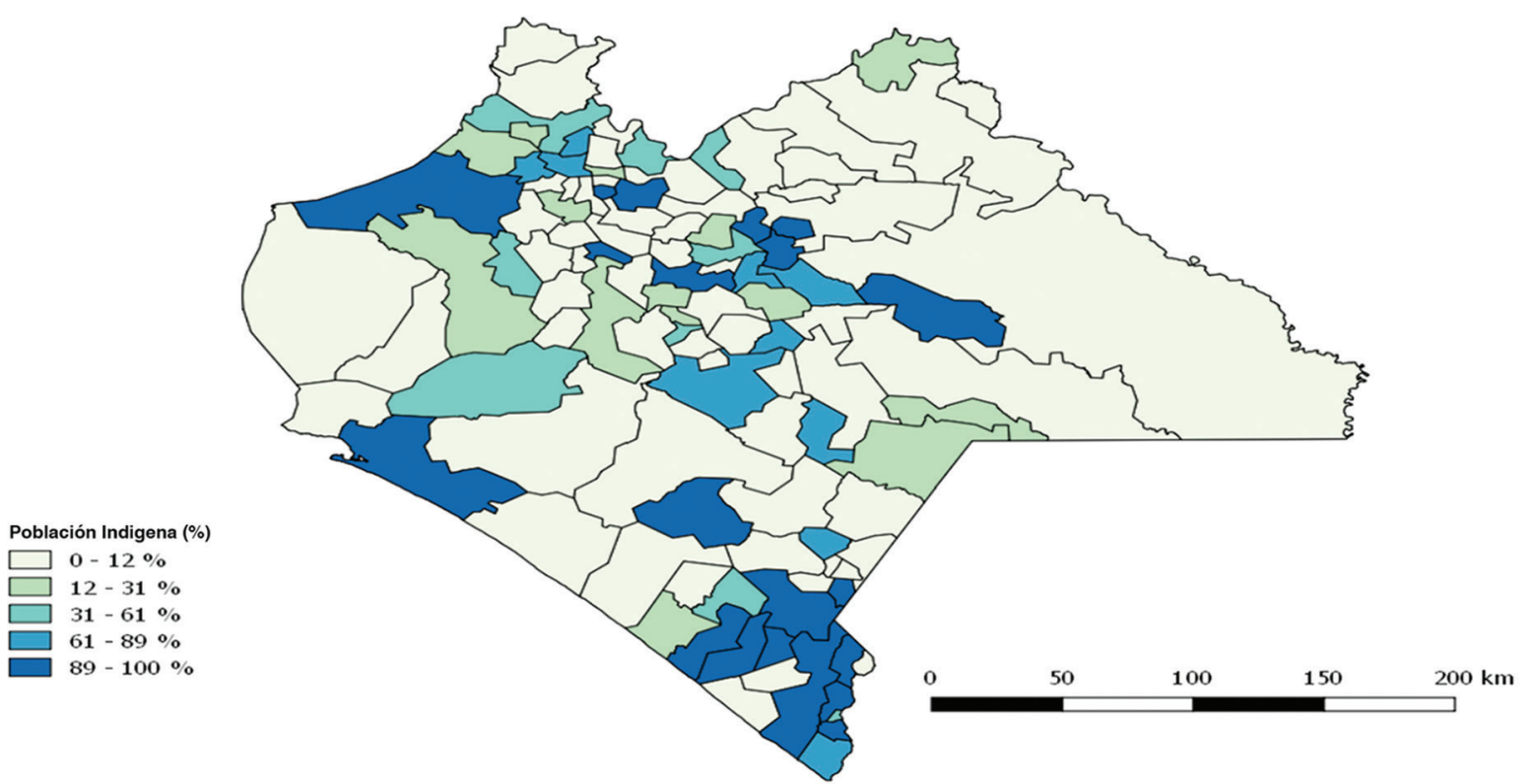

Figura 5. Mapa de la distribución de la población por porcentaje de población indígena por municipio, en Chiapas. Cartografía de municipios y clasificación por quintiles de población indígena.

Los resultados de la aplicación y visualización del índice LISA mostrados en la figura 6 permiten rechazar la hipótesis de no asociación espacial con respecto a la malaria. Los cuadrantes alto-alto indicaron cuatro conglomerados espaciales en donde los municipios tuvieron alta incidencia de malaria, rodeados de municipios vecinos con también alta incidencia de malaria. Esta determinación permitió observar los epicentros de la transmisión de la malaria en Chiapas. Los SIG y las métricas geoepidemiológicas ofrecen mejores oportunidades y mayor precisión para la identificación de grupos y áreas de alto riesgo epidemiológico, así como de los principales determinantes de dicho riesgo epidemiológico. 


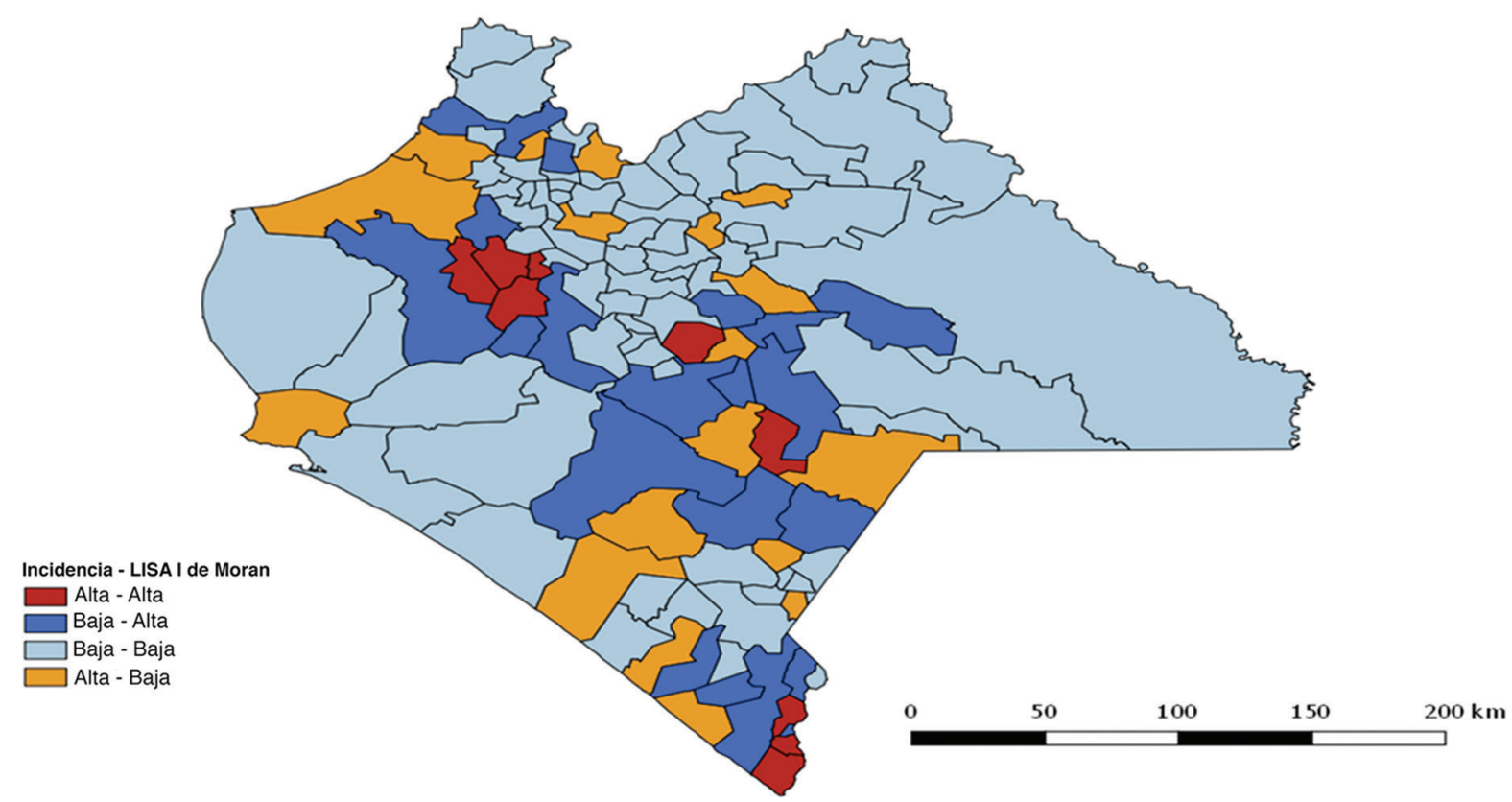

Figura 6. Mapa de la autocorrelación espacial usando el índice LISA de la malaria por municipios, en Chiapas, México. Visualización de la autocorrelación espacial de la malaria usando LISA para la detección de conglomerados estadísticamente significativos de malaria. Los conglomerados en rojo indican municipios con alta incidencia de malaria y con municipios vecinos también con alta incidencia de malaria.

\section{Discusión y conclusiones}

Este análisis geoepidemiológico proporciona una mejor caracterización espacial de la distribución epidemiológica de la malaria en Chiapas durante los años estudiados, así como de los factores de riesgo que se asocian en el nivel ecológico-poblacional con el incremento del riesgo epidemiológico de malaria. Se logró visualizar, a través de los mapas temáticos y las herramientas analíticas de los SIG, que los factores de riesgo poblacional identificados como asociados estadísticamente a la malaria en Chiapas fueron la baja tasa de alfabetización, la presencia de redes específicas de carreteras que permiten una mayor difusión espacial de la malaria, y el alto porcentaje de población indígena que refleja las desigualdades sociales en salud y la gran carga de enfermedad que este grupo tiene. Así mismo, de especial relevancia fue la identificación de un importante conglomerado espacial de malaria en proximidad a áreas y población de la frontera sur de Chiapas.

Las herramientas y métodos geoepidemiológicos pueden tener un papel importante en las actividades habituales ${ }^{1,20,40,41}$ de vigilancia epidemiológica de la malaria en Chiapas, así como de otros problemas prioritarios de salud.

El uso de los conglomerados espaciales ofrece información valiosa para poder conformar los distintos estratos locales de riesgo de malaria y permitir una estratificación epidemiológica operacional que apoye la selección de medidas e intervenciones más efectivas y equitativas para la prevención y el control de la malaria.

Se recomienda que los estudios epidemiológicos y la estratificación epidemiológica de la transmisión de la malaria en Chiapas y otras áreas críticas de malaria utilicen un enfoque epidemiológico y multidisciplinario en su ejecución, considerando que la distribución epidemiológica, la densidad y la difusión espacial de los casos de malaria pueden estar influenciadas localmente de manera diferencial por la forma en que en el nivel local se expresan los principales factores de riesgo culturales, económicos, políticos, de migración irregular y de la organización inadecuada de los servicios de salud.

\section{Agradecimientos}

Mi especial agradecimiento a los doctores Germán E. Fajardo Dolci, Luis G. Durán Arenas, Gustavo Olaiz Fernández y Malaquías López Cervantes por su apoyo para el fortalecimiento de la colaboración académica con el Global Public Health Observatory que dirijo en la Bloomberg School of Public Health, de la Universidad Johns Hopkins. Especial reconocimiento para la Dra. Reyna Lizette Pacheco, la Lic. Maricarmen García 
Franco y Thelma Alcántara Ayala, del Centro de Investigación en Políticas, Población y Salud de la Facultad de Medicina de la Universidad Nacional Autónoma de México por el importante apoyo brindado durante el proceso de admisión a la Academia Mexicana de Medicina. Mi agradecimiento personal y profesional al Lic. Manuel Vidaurre, quien durante varios años me ha brindado un importante apoyo metodológico, especialmente en el desarrollo de las aplicaciones y estadísticas geoespaciales requeridas en la epidemiología profesional y la salud pública; así mismo, por su colaboración como coinstructor de mi curso Epidemiologic Applications of GIS, que se ofrece por más de una década en la Johns Hopkins Bloomberg School of Public Health y en donde ejecutamos diversos análisis geoespaciales con la información incluida en esta investigación ${ }^{31}$.

\section{Bibliografía}

1. Castillo-Salgado, C. Trends and directions of global public health surveillance. Epidemiol Rev. 2010;32:93-109.

2. Oliveira MA, Ribeiro H, Castillo-Salgado C. Geospatial analysis applied to epidemiological studies of dengue: a systematic review. Rev Bras Epidemi2013;16:907-17.

3. Rushton G. Public health, GIS, and spatial analytic tools. Ann Rev Public Health. 2003;24:43-56.

4. Martin C, Curtis B, Fraser C, et al. The use of a GIS-based malaria information system for malaria research and control in South Africa. Health Place. 2002;8:227-36

5. Rodríguez MH. Control focal del paludismo en México. Fifth Meeting of the Steering Committee. Regional Program of Action and Demonstration of Sustainable Alternatives for Malaria Control without Use of DDT in Mexico and Central America. DDT/UNEP/GEF/PAHO PROJECT. July $1-2,2008$.

6. Betanzos-Reyes AF, Rodríguez MH, Durán-Arenas LG, et al. Comparative analysis of two alternative models for epidemiological surveillance in the Mexican Malaria Control Program. Health Policy. 2007;80:465-82.

7. Betanzos Reyes AF. La malaria en México. Progresos y desafíos hacia su eliminación. Bol Med Hosp Infant Mex. 2011;68:159-68.

8. Castillo-Salgado C. Role and strategies of surveillance networks in handling emerging and reemerging viral infections. En: Singh SK, editor. Vira infections and global change. Hoboken, NJ: John Wiley \& Sons; 2013. p. 215-16.

9. CDC (2017). GIS and public health at CDC. (Consultado el 22/8/2017.) Disponible en: https://www.cdc.gov/gis/index.htm

10. Castillo-Salgado C, Vidaurre M, Martinez R, et al. Sistemas de información geográfica. Conceptos básicos. Washington, DC: Paltex. Organización Panamericana de la Salud; 2002.

11. Cromley EK, McLafferty SL. GIS and public health. New York: The Guilford Press; 2002. p. 62-190.

12. Lozano-Fuentes S, Elizondo-Quiroga D, Farfan-Ale JA, et al. Use of Google Earth to strengthen public health capacity and facilitate management of vector-borne diseases in resource-poor environments. Bull World Health Organ. 2008;86:718-72.

13. WHO. Global technical strategy for malaria 2016-2030. Geneva: WHO; 2015. p. 1-35. Disponible en: http://apps.who.int/iris/bitstream/10665/ 176712/1/9789241564991_eng.pdf?ua=1

14. WHO Global Malaria Programme. 2016 World Malaria Report. Geneva: WHO; 2016.
15. Alonso PL, Brown G, Arevalo-Herrera $\mathrm{M}$, et al. A research agenda to underpin malaria eradication. PLoS Med. 2011;8:e1000406.

16. The Malaria Eradication Research Agenda (malERA). Disponible en: http://collections.plos.org/malera

17. Castillo-Salgado C. Estratificación epidemiológica de la malaria en las Américas. Boletín Epidemiológico de la OPS. 1991;12(4). Washington DC; diciembre 1991. Disponible en: http://cidbimena.desastres.hn/docum/ops/publicaciones/Epidemiologico/BE v12n4.pdf

18. Castillo-Salgado C. Epidemiological risk stratification of malaria in the Americas. Mem Inst Oswaldo Cruz. 1992;87(Suppl 3):115-20.

19. Castillo-Salgado $\mathrm{C}$, Bayona Celis $\mathrm{M}$. Use of epidemiological research in the development of the epidemiological risk strata and the selection of control interventions. Training Modules. Baltimore MD and Washington DC; 2012.

20. Canelas T, Castillo-Salgado $\mathrm{C}$, Ribeiro H. Systematized literature review on spatial analysis of environmental risk factors of malaria transmission. Adv Infect Dis. 2016;6:52-62.

21. Kelly GC, Tanner M, Vallely A, et al. Malaria elimination: moving forward with spatial decision support systems. Trends Parasitol. 2012·28:297-304.

22. Kleinschmidt I, Bagayoko M, Clarke GP, et al. A spatial statistical approach to malaria mapping. Int J Epidemiol. 2000;29:355-61.

23. Valle D, Tucker Lima JM. Large-scale drivers of malaria and priority areas for prevention and control in the Brazilian Amazon region using a novel multi-pathogen geospatial model. Malar J. 2014;13:443.

24. Brooker S, Leslie T, Kolaczinski K, et al. Spatial epidemiology of Plasmodium vivax, Afghanistan. Emerg Infect Dis. 2006;12:1600-2.

25. PAHO/WHO. Regional Program of Action and Demonstration of Sustainable Alternatives to DDT for Malaria Vector Control in Mexico and Central America. Disponible en: http://www.paho.org/hq/index.php?option=com_content\&view=article\&id=2712\&ltemid=2172\&lang=en

26. QGIS 2.10. Free and open source GIS. Disponible en: http://www.qgis. org/en/site.

27. Moran's I. Definition and uses. Disponible en: https://en.wikipedia.org/ wiki/Moran\%27s_l

28. Anselin L. Computing environments for spatial data analysis. J Geogr Syst. 2000;2:201-20.

29. Anselin L. Local indicators of spatial autocorrelation: LISA. Geographical Analysis. 1995;27:93-115.

30. Vidaurre M, Castillo-Salgado C. GeoPublicHealth. Disponible en: https:// github.com/ePublicHealth/GeoPublicHealth

31. Bloomberg School of Public Health. Johns Hopkins University. Graduate Course: Epidemiologic applications of GIS. CoursePlus Syllabus. Baltimore, MD. Disponible en: https://courseplus.jhu.edu/core/index.cfm/go/ course.home/coid/8236

32. Moonen B, Cohen JM, Snow RW, et al. Operational strategies to achieve and maintain malaria elimination. Lancet. 2010;376:1592-603.

33. Alimi TO, Fuller DO, Herrera SV, et al. A multi-criteria decision analysis approach to assessing malaria risk in northern South America. BMC Public Health. 2016;16:1-10.

34. Larocca A, Moro V R, Marconi M. Malaria diagnosis and mapping with $\mathrm{m}$-Health and geographic information systems (GIS): evidence from Uganda. Malar J. 2016;15:520.

35. Martin C, Curtis B, Fraser C, et al. The use of a GIS-based malaria information system for malaria research and control in South Africa. Health \& Place. 2002;8:227-36.

36. Martens $P$, Hall L. Malaria on the move: human population movement and malaria transmission. Emerg Infect Dis. 2000;6:103-9.

37. Recht J, Siqueira AM, Monteiro WM, et al. Malaria in Brazil, Colombia, Peru and Venezuela: current challenges in malaria control and elimination. Malar J. 2017;16:273.

38. Herrera S, Ochoa-Orozco SA, González IJ, et al. Prospects for malaria elimination in Mesoamerica and Hispaniola. PLoS Negl Trop Dis. 2015;9:e0003700.

39. Martin C, Curtis B, Fraser C, et al. The use of a GIS-based malaria information system for malaria research and control in South Africa. Health Place. 2002;8:227-36.

40. Sipe NG, Dale $P$. Challenges in using geographic information systems (GIS) to understand and control malaria in Indonesia. Malar J. 2003;2:36.

41. Jajosky RA, Groseclose SL. Evaluation of reporting timeliness of public health surveillance systems for infectious diseases. BMC Public Health. 2004:4:29. 